\title{
Crystallographic study on estimation of the valence of each of the four Mn atoms in Photosystem II using anomalous diffraction techniques
}

\author{
Yasufumi Umena ${ }^{\mathrm{a}}$, Keisuke Kawakami ${ }^{\mathrm{b}}$, Nobuo Kamiya ${ }^{\mathrm{b}}$, Yoshiaki Kawano ${ }^{\mathrm{c}}$, Keitaro \\ Yamashita $^{\mathrm{d}}$, Hideo $\mathrm{Ago}^{\mathrm{c}}$, Masaki Yamamoto ${ }^{\mathrm{c}}$, Jian-Ren Shen ${ }^{\mathrm{a}, \mathrm{e}}$ \\ ${ }^{a}$ Research Institute for Interdisciplinary Science, Okayama University, 3-1-1 Tsushima-naka, \\ Kita-ku, Okayama 700-8530, JAPAN. umena@okayama-u.ac.jp \\ ${ }^{b}$ The OCU Advanced Research Institute for Natural Science and Technology, Osaka City \\ University, 3-3-1 Sugimoto, Sumiyoshi-ku, Osaka 558-8585, JAPAN \\ 'RIKEN SPring-8 Center, 1-1-1 Kouto, Sayo-cho, Sayo-gun, Hyogo 679-5148, JAPAN \\ ${ }^{\mathrm{d}}$ Department of Biological Sciences, Graduate School of Science, The University of Tokyo, \\ Hongo, Bunkyo-ku, Tokyo 113-0033, JAPAN \\ ${ }^{\mathrm{e}}$ Graduation School of Natural Science and Technology, Okayama University, 3-1-1 Tsushima- \\ naka, Kita-ku, Okayama 700-8530, JAPAN
}

Photosystem II (PSII) in photosynthetic organisms catalyzes light-induced water oxidation leading to the production of protons, electrons and molecular oxygen. PSII is a multi-subunit membrane protein complex with a total molecular weight of $350 \mathrm{kDa}$ in a monomer. We have reported the cyanobacterial PSII structure at a resolution of $1.9 \AA$ prepared from Thermosynechococcus vulcanus (PDB: $3 \mathrm{WU} 2)^{[1]}$. The catalytic center of oxygen-evolving complex (OEC) in PSII is composed of four $\mathrm{Mn}$ atoms and one $\mathrm{Ca}$ atom organized in a $\mathrm{Mn}_{4} \mathrm{CaO}_{5^{-}}$ cluster, which cycles through several different redox states to accomplish the catalytic process. The OEC has been known as the mixed valent four Mn cluster by various analysis. We determined the positions of the atoms in OEC using the electron density map for the first time. However, the valences of each of the four $\mathrm{Mn}$ atoms in OEC and their participation in the redox reactions are still under debates. In order to uncover the catalytic mechanism by OEC, it is important to determine the valence of each of these Mn atoms as well as to solve the detailed structure.

In this study, we analyzed the individual valence of $\mathrm{Mn}$ atoms in OEC by X-ray crystallographic analysis using the anomalous diffraction technique at the wavelength of Mn Kabsorption edge. It is well-known that the chemical shift of $\mathrm{K}$-absorption edge occurs depending on the oxidation state of a transition metal atom, but the intensity of the anomalous scattering also changes according to the oxidation state of the metal atom. In this method, we sliced the PSII crystals into cylinder-shaped ones with the same thickness in the whole oscillation angle using a deep-UV pulsed laser ${ }^{[2]}$, in order to suppress the fluctuation of X-ray absorption effects due to different thickness of the crystals. The similar thickness of crystals used also improved homogeneity of the partial data collected using multi-crystals. We collected diffraction data at the wavelength of Mn K-absorption edge (1.892 $\AA$ ), which was determined from a PSII crystal, and calculated the anomalous difference Fourier map (anomalous map) corresponding to each $\mathrm{Mn}$ atom in OEC. We found differences in the intensities of the anomalous maps for each of the four Mn atoms in OEC, indicating differences in the oxidation states among each of the four Mn atoms. In order to avoid possible X-ray reduction of OEC and to verify the threshold dose that causes the $\mathrm{X}$-ray reduction, we collected $28 \mathrm{X}$-ray datasets using different X-ray doses ranging from $7 \mathrm{kGy}$ to $9.5 \mathrm{MGy}$, and compared the intensities of the anomalous maps obtained for OEC. We will discuss the valence distribution of each of the four $\mathrm{Mn}$ atoms in OEC while taking into consideration the influence of X-ray dose and demonstrate the usability for the simultaneous analysis of structure and valence in a multi-metal cluster by this anomalous diffraction technique.

\section{References}

[1] Y. Umena, K. Kawakami, J.-R. Shen, N. Kamiya. (2011). Nature, 473, 55-60

[2] H. Kitano, H. Adachi, A. Murakami, et al. (2004). Jpn. J. Appl. Phys., 44, L54 